\title{
Review of Estimation on Vertex Normal Vector and Discrete Curvature of Triangular Mesh
}

\author{
Ting $\mathrm{Wu}^{1, \mathrm{a}}$ and Libing Zhang ${ }^{1, \mathrm{~b}}$ \\ ${ }^{1}$ College of Mechanical and Electrical Engineering, Jiaxing University, Jiaxing, Zhejiang, China \\ awuting628@163.com, ${ }^{b}$ libinzhan@126.com
}

Keywords: Triangular mesh; vertex normal vector; discrete curvature; Gaussian curvature; mean curvature.

Abstract. In order to reconstruct the gingival margin curve of the discrete curvature estimation of triangular mesh surfaces, the estimation methods of the vertex normal vector and discrete curvature of the triangular mesh model are summarized and reviewed. By the analysis of the existing method of vertex normal vector and discrete curvature for triangular mesh, estimation calculation of vertex normal vector mainly has three kinds of methods, where the advantages and disadvantages are compared, and estimation methods of discrete curvature for triangular mesh mainly have a local surface fitting, Laplace-Beltrami operator, Moreton and Sequin, and so on. By the review, a more appropriate method is sought for the reconstruction of gingival margin curve.

\section{Introduction}

In the dental digital system, the 3D digital dental model is obtained by using the optical measurement technique, and the curved surfaces of the 3D model are expressed in the form of triangular mesh. Curvature is an important feature of a curved surface, and the calculation of curvature has some perfect and mature methods for the surface expressed in parametric form [1]. However, these traditional methods are not suitable to solve the curvature of the surface with discrete representation. The 3D digital dental model is represented by triangular mesh, and the discrete curvature estimation of triangular mesh surface needs to be solved during the reconstruction of the gingival margin curve. The vertex normal vector of the triangular mesh is calculated during the discrete curvature of triangular mesh surface. Therefore, the estimation methods of the vertex normal vector and discrete curvature of the triangular mesh model are analyzed and reviewed in this paper.

\section{Estimation on Vertex Normal Vector of Triangular Mesh}

Triangular Mesh Model. Assuming that $V_{0}$ is a vertex of triangular mesh and $V_{i}(\mathrm{i}=1,2, \ldots, m)$ are connection points of $V_{0} . n_{i}(\mathrm{i}=1,2, \ldots, m)$ are unit normal vectors of triangular patch, which are shown in Figure 1. The normal vector $n_{i}$, which is the normal vector of the triangle patch composed of $V_{0}, V_{i}$ and $V_{i+1}$, can be expressed as follows:

$$
n_{i}=\frac{\left(V_{i}-V_{0}\right) \times\left(V_{i+1}-V_{0}\right)}{\left\|\left(V_{i}-V_{0}\right) \times\left(V_{i+1}-V_{0}\right)\right\|} \quad i=1,2, \mathrm{~L}, m
$$

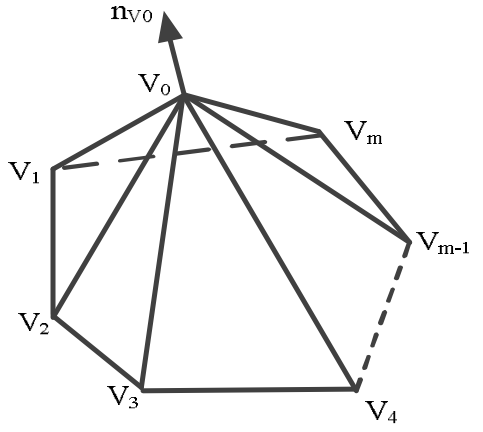

Fig. 1 The normal vector of triangular mesh model 
Estimation on Vertex Normal Vector of Triangular Mesh Model. For calculation of the vertex normal vector for the triangle mesh model, the some methods are mainly used as following:

(1) By using the area of each triangle patch for the triangle mesh model as the weight, the unit normal vector of each triangle patch is weighted average, and the vertex normal vector of the triangular mesh model is obtained [2], which can be written as follows:

$$
n_{V_{0}}=\frac{\sum_{i=1}^{m} A_{i} n_{i}}{\left\|\sum_{i=1}^{m} A_{i} n_{i}\right\|}
$$

where $n_{V_{0}}$ is the vertex normal vector of the triangular mesh model, $n_{i}$ is the normal vector of the triangle patch, and $A_{i}$ is the area of the triangle patch.

The area of the triangle patch can be expressed as follows:

$$
A_{i}=\frac{\left(V_{i}-V_{0}\right) \times\left(V_{i+1}-V_{0}\right)}{2} \quad i=1,2, \mathrm{~L}, m
$$
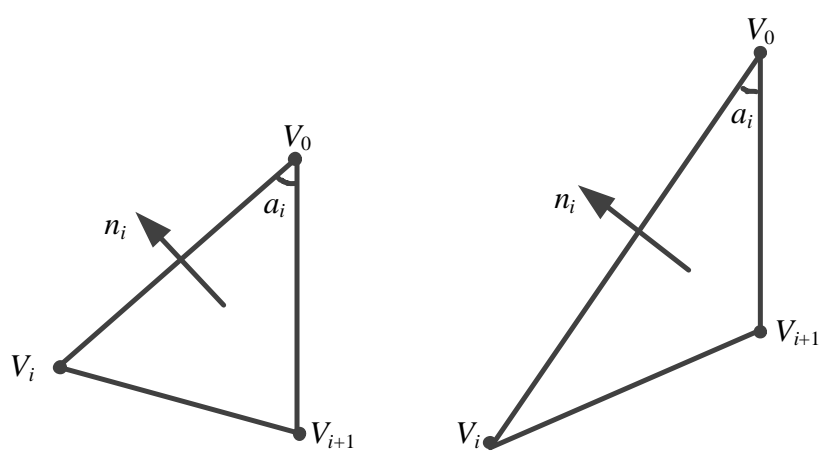

Fig. 2 The same area and normal vector, and the different shape of the triangle patch

The area of the two triangle patches is the same, however, the angle of the triangle patches is different, which may lead to a large difference in the shape (Fig. 1). Therefore, the Eq. (2) does not reflect the influence of the shape of the triangular patch on the vertex normal vector of the triangular mesh model.

(2) In order to reduce the influence of triangle angle on the vertex normal vector of the triangular mesh model, the unit normal vector of each triangle patch is weighted average by using the area and the angle of each triangle patch for the triangle mesh model as the weight [3], and the vertex normal vector of triangular mesh model is obtained, which can be written as follows:

$$
n_{V_{0}}=\frac{\sum_{i=1}^{m} A_{i} \alpha_{i} n_{i}}{\left\|\sum_{i=1}^{m} A_{i} \alpha_{i} n_{i}\right\|}
$$

where $\alpha_{i}$ is an angle between the two sides of the vertex $V_{0}$.

(3) Position relation of three vertices of the triangle patch should been shown during the vertex normal vector calculation in the triangular mesh model, and the role of vertices is highlighted. So the unit normal vector of each triangle patch is weighted average by using the distance from the center to the vertex, and the angle of each triangle patch for the triangle mesh model as the weight [4], and the vertex normal vector of the triangular mesh model is obtained, which can be written as follows:

$$
n_{V_{0}}=\frac{\sum_{i=1}^{m} B_{i} \alpha_{i} n_{i}}{\left\|\sum_{i=1}^{m} B_{i} \alpha_{i} n_{i}\right\|}
$$


where $B_{i}$ is the distance from the center of the triangle patch to the vertex.

\section{Discrete Curvature Estimation of Triangular Mesh}

For the traditional parametric surfaces and implicit surfaces, the calculation of the micro components has a mature theory and method. However, the triangular mesh is a piecewise linear surface, and a classical differential geometry does not provide sufficient theory for the computation of discrete triangular mesh surfaces. At present, the discrete curvature is commonly used calculation methods of a local surface fitting, Laplace-Beltrami operator, Moreton and Sequin, and so on.

Local Surface Fitting Method. A pair of orthogonal vectors $u$ and $v$ is selected on the plane of the normal vector for triangular mesh vertex, and a local coordinate system is established at the vertex of the triangular mesh. For example, the local surface fitting method uses the parabolic surface, which can be written as follows [5]:

$$
S(u, v)=a u^{2}+b u v+c v^{2}
$$

The parabolic surface is fitted by using least square method, and the objective function can be expressed as follows:

$$
J_{\min }=\sum_{i=1}^{k}\left[h_{i}-\left(a u_{i}^{2}+b u_{i} v_{i}+c v_{i}^{2}\right)\right]^{2}
$$

The maximum and minimum principal curvatures of the vertex of triangular mesh are solved, which can be written as follows:

$$
\left\{\begin{array}{l}
k_{\max }=a+c+\sqrt{(a-c)^{2}+b^{2}} \\
k_{\min }=a+c-\sqrt{(a-c)^{2}+b^{2}}
\end{array}\right.
$$

Laplace-Beltrami Operator. Laplace-Beltrami operator $(\Delta=H n)$ and mean curvature manifold of a surface of the triangular mesh are introduced into the estimation method of discrete Curvature [6]. The estimation method has three forms, namely Taubin method, Mayer method, and Desbrun method.

(1) In Taubin method, Laplace-Beltrami operator is discrete, which is expressed as follows [7]:

$$
\Delta=\sum_{j \in N(i)} w_{i j}\left(p_{j}-p_{i}\right)
$$

where $p_{i}$ and $p_{j}$ are point on the mesh surface, the weighted factor $w_{i j}$ satisfies $\sum_{j \in N(i)} w_{i j}=1$.

$$
w_{i j}=\frac{\tau\left(p_{i}, p_{j}\right)}{\sum_{k \in N(i)} \tau\left(p_{i}, p_{k}\right)}
$$

where $\tau\left(p_{i}, p_{j}\right)$ is the total area of triangles of the edge $p_{i} p_{j}$.

(2) In Mayer method, Laplace-Beltrami operator is integrated, which can be written by the Green formula in differential geometry,

$$
\int_{D} \Delta f(x) d x=\int_{\partial D} \partial f(s) d s
$$

(3) Desbrun method

Moreton and Sequin Method. By using the Euler theorem of differential geometry, the relationship between the curvature of the surface method, principal curvature, and principal direction is established $[6,8]$. The average value of each normal vector of the triangular patches around the vertex $v_{i}$ is the normal vector of the triangular mesh surface at the vertex $v_{i}$, which is expressed as $n$. The plane through the vertex $v_{i}$ and the plane perpendicular to the normal vector $n$ is called the tangent plane of the mesh surface, $t_{j}$ is the unit projection of the vector $v_{i} v_{j}$ in the tangent plane of the mesh surface, and the curvature of the curved surface at the vertex $v_{i}$ along $v_{i} v_{j}$ direction can be expressed as follows: 


$$
k_{j}=\frac{2 n \cdot\left(v_{j}-v_{i}\right)}{\left(v_{j}-v_{i}\right) \cdot\left(v_{j}-v_{i}\right)}
$$

Assuming that $b_{x}$ and $b_{y}$ are a set of bases on the tangent plane of the mesh surface, $t_{j x}$ and $t_{j y}$ are the coordinates in $b_{x}$ and $b_{y}$, and $e_{x}$ and $e_{y}$ are the coordinates of the principal direction in $b_{x}$ and $b_{y}$. According to Euler theorem, the expression is written as follows:

$$
\begin{aligned}
& k_{j}=\left(\begin{array}{c}
t_{j x} \\
t_{j y}
\end{array}\right)^{T} \cdot K \cdot\left(\begin{array}{c}
t_{j x} \\
t_{j x}
\end{array}\right) \\
& K=\left[\begin{array}{cc}
e_{x} & e_{y} \\
-e_{y} & e_{x}
\end{array}\right] \cdot\left[\begin{array}{cc}
k_{1} & 0 \\
0 & k_{2}
\end{array}\right] \cdot\left[\begin{array}{cc}
e_{x} & e_{y} \\
-e_{y} & e_{x}
\end{array}\right]^{-1}, i, j=1,2, \mathrm{~L}, m, \text { and the equations are obtained, which can }
\end{aligned}
$$

be written as follows: $A x=b$, where

$$
A=\left[\begin{array}{ccc}
t_{1 x} t_{1 x} & t_{1 x} t_{1 y} & t_{1 y} t_{1 y} \\
t_{2 x} t_{2 x} & t_{2 x} t_{2 y} & t_{2 y} t_{2 y} \\
\mathrm{M} & \mathrm{M} & \mathrm{M} \\
t_{m x} t_{m x} & t_{m x} t_{m y} & t_{m y} t_{m y}
\end{array}\right], b=\left[\begin{array}{c}
k_{1} \\
k_{2} \\
\mathrm{M} \\
k_{m}
\end{array}\right] \text {, and } x=\left[\begin{array}{c}
x_{0} \\
x_{1} \\
x_{2}
\end{array}\right]=\left[\begin{array}{c}
e_{x}^{2} k_{1}+e_{y}^{2} k_{2} \\
e_{x} e_{y}\left(k_{1}-k_{2}\right) \\
e_{x}^{2} k_{2}+e_{y}^{2} k_{1}
\end{array}\right] \text {. So Mean curvature and }
$$

Gauss curvature can be obtained, which can be written as follows:

$$
K_{H}=\frac{x_{0}+x_{2}}{2}, K G=2 x_{0} x_{2}-\frac{x_{1}^{2}}{2}
$$

\section{Conclusions}

In this paper, a conclusion is drawn that estimation calculation of vertex normal vector mainly has three kinds of methods, and estimation methods of discrete curvature for triangular mesh mainly have the local surface fitting, Laplace-Beltrami operator, Moreton and Sequin, and so on. The advantages and disadvantages of those methods are summarized and compared, and a more appropriate method is sought for the reconstruction of gingival margin curve by the analysis of the existing method of vertex normal vector and the discrete curvature for triangular mesh.

\section{Acknowledgements}

The work was financially supported by the Zhejiang Natural Science Foundation of (LQ14E050006), and the National Natural Science Foundation of China (51405197).

\section{References}

[1] C. Dong, G. Wang: J Zhejiang Univ Sci Vol. 6A (2005), p. 128

[2] D. L. Page, A. Koschan, Y. Sun: Proceedings of the International Conference on Computer Vision and Pattern Recognition 2001, San Francisco ( 2001), p. 162

[3] H. Shen, J. Li, L. Zhou: Computer Engineering and Applications Vol. 41(2005), p. 12

[4] S. Yan, L. Sun, N. Ji, A. Yang: Journal of Hebei Polytechnic University (Natural Science Edition) Vol. 30 (2008), p. 104

[5] T. Wu, W. Liao, N. Dai: China Mechanical Engineering Vol. 23 (2012), p. 2245

[6] H. Fang, G. Wang: Journal of Conputer-Aided Design \& Computer Graphics Vol. 17 (2005), p. 2500

[7] S. Wu, C. Wang: Chinese Journal of Mechanical Engineering Vol. 43 (2007), p. 230

[8] X. Ma, X. Xie: Computer Engineering and Applications Vol. 50 (2014), p. 196 